\title{
Calculating the predictive power of the Henson field screener in a population at risk of glaucomatous field loss
}

\author{
Stephen A Vernon, Donald J Henry, Susan J Jones
}

\begin{abstract}
Suprathreshold field screeners are in common use for the detection of glaucomatous field loss. The predictive power of a positive result $(\mathbf{P P}+)$ depends on the sensitivity and specificity of the screener in the population in which it is to be used. Using data from 755 normal individuals (1510 eyes), we calculated the PP+ of the Henson CFS2000 screening programme for a population aged 50 and over. $4.3 \%$ of normal eyes failed the screening programme. Ignoring one or two misses on the screening programme immediately adjacent to the disc reduced this figure to $1.3 \%$ and significantly improved the PP+ of the programme. Calculations of the PP+ at increasing glaucoma prevalence levels indicates this to be particularly relevant at low levels such as those encountered when screening middle aged and elderly populations. Optometrists should perform routine field analysis when screening for glaucoma provided they adhere to strict protocols.
\end{abstract}

The 26-point Henson CFS2000 screening programme has been formulated to provide maximum sensitivity and specificity when screening for glaucomatous field loss. In a study on $\mathbf{9 4}$ suspect eyes the programme's sensitivity and specificity were calculated to be $90 \%$ and $88 \%$ respectively. ${ }^{1}$ If an eye fails the screening programme, the operator is required to 'extend', testing a total of 66 or 132 points. This might appear to suggest that $12 \%$ of 'normal' eyes will require an extended field.

Sensitivity, a measure of the false negatives, and specificity, a measure of the false positives, are dependent on the certainty that a subject is positive or negative for the condition and on the prevalence of the disease in the population in which the test is to be used. Neither of these parameters of the efficiency of a test can be extrapolated from a small group of patients under suspicion to the general population at risk from glaucoma. Although the sensitivity of the test can be estimated when the prevalence of the disease is high in a sample, the specificity cannot.

In a recent study of the methods employed by optometrists when screening for glaucoma almost $80 \%$ used visual field analysis, though usually only when either the disc appeared abnormal or the intraocular pressure (IOP) was raised. ${ }^{2}$

In practice, when screening an individual for glaucomatous field loss, the optometrist needs to know the predictive power of a positive test $(\mathrm{PP}+) . \mathrm{It}$ is the chance that a positive result indicates a true positive identification of the disease process and is related to both sensitivity and specificity.

To calculate the predictive power of the screening programme on the Henson CFS2000 in a population, the following data are required from that population: $(a)$ the incidence of screen failures in normal persons; $(b)$ the prevalence of field defects from other causes; $(c)$ the prevalence of glaucomatous field loss; $(d)$ the false negative rate on screening eyes with field loss from glaucoma.

If $(c)$ remains a variable, the $\mathrm{PP}+$ for each value of $(c)$ can be calculated only if $(a),(b)$, and $(d)$ are known. (d) has been determined to be $10 \%$ in a sample of eyes representative of those presenting to a hospital department with glaucomatous field.loss. ${ }^{1}$

Therefore in order to estimate the predictive power of the Henson CFS2000 screening programme in the field we analysed the data on normal persons and patients with non-glaucomatous field defects identified on screening a general practice population aged 50 and over.

\section{Material and methods}

The population of a general practice aged 50 and over were invited by letter to attend for glaucoma screening: 874 people $(88.5 \%$ of those invited) attended. They were screened by the following tests: intraocular pressures with a non-contact tonometer, optic disc assessment by an experienced ophthalmologist, and visual field analysis. The only exclusions from screening were a history of dementia, glaucoma, or blind registration. Computer analysis of the practice records revealed no significant difference between those who did and did not attend when the following variables were examined: age, sex, family history of glaucoma, known hypertension, or known diabetes.

Intraocular pressures were measured with the Keeler Pulsair non-contact tonometer; the mean of four readings per eye was taken. Visual field analysis was then performed with the Henson CFS2000 as described below, followed by optic disc assessment. All eyes were examined by a single ophthalmologist (SAV) with a narrow beam direct ophthalmoscope through undilated pupils, discs being graded 'blind' as normal or suspicious. Any patient with an IOP $>22 \mathrm{mmHg}$ or having a suspicious optic disc was referred for a hospital assessment to determine the true status of the eye.

The visual fields were examined in the following manner with the screening programme of the Henson CFS2000: 
Figure 1: Predictive power of a positive result on the Henson screening programme at different prevalence levels of glaucoma. Filled boxes determine the levels when one or two misses by the blind spot are considered insignificant.
Figure 2: Predictive power of $a$ positive result on the Henson screening programme at different prevalence levels of glaucoma when other ophthalmoscopically detectable causes of field loss are excluded. Filled boxes indicate the levels when one or two misses by the blind spot are considered insignificant.

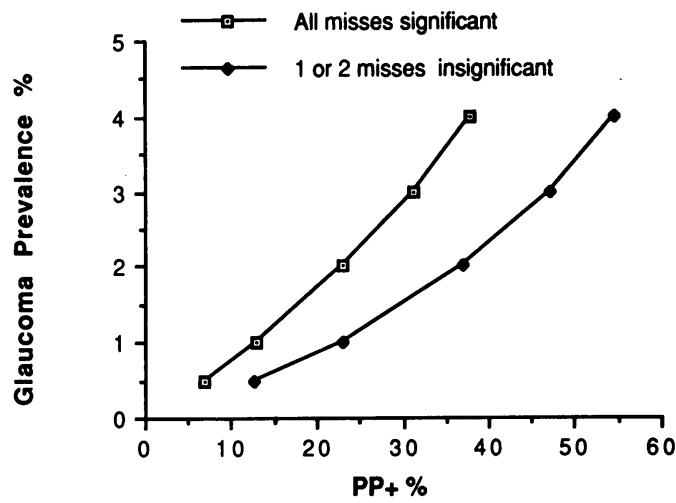

The test was performed either with single vision reading spectacles or unaided if these were not available. The right eye was always tested first. The minimum vision required to perform the test was defined as the ability to see the central target on the screen at $1 / 3$ metre following the inbuilt ambient lighting check on the Henson apparatus. Central thresholds were then determined for each eye, threshold being taken as the lowest illumination required to detect $50 \%$ of the central targets in at least two of three presentations. The 26-point suprathreshold screening programme was then performed at $0.5 \mathrm{log}$ unit above threshold. Three attempts were made at each setting before an eye was deemed to have missed a point. Any missed point on this test required the operator to extend to a 66-point test. Any eye which recorded a 'suspicious' or 'defective' field on the internal scale was classified as pathological.

Any eye that required an extended test was carefully examined to determine the presence of coexisting pathology that could have resulted in a screening failure.

Results are presented in terms of eyes rather than patients because Henson's data reflecting the false negative rate for the screening programme relate to eyes only. However, only patients in whom both eyes were normal on all accounts could contribute to the normal data.

Calculations of predictive powers assume a $90 \%$ sensitivity for the screening programme. ${ }^{1}$

\section{Results}

The number of eyes attempting the field test was 1748 (874 patients, mean age 65 years). Twentyfive failed to detect the central target due to poor central acuity; therefore 1723 eyes were tested.

One hundred and thirteen eyes $(6.56 \%)$ from 98 patients (mean age 65.2 years) required an extended test. Seven were diagnosed as suffering

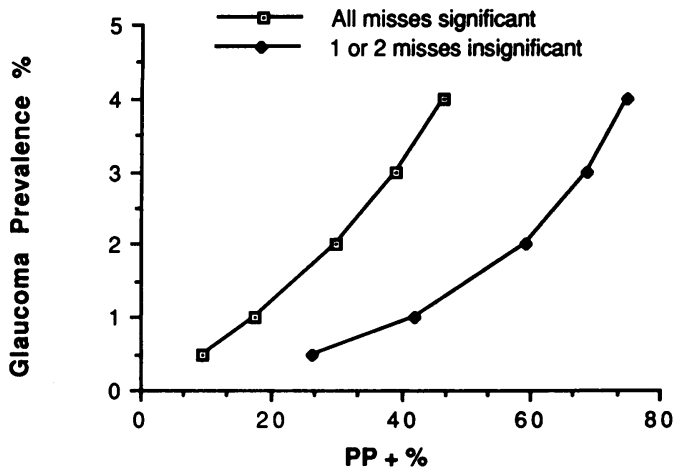

from glaucoma at the hospital assessment and $106(6 \cdot 15 \%)$ were either normal or had other pathology. The $95 \%$ confidence limits (CL) on this percentage are $5 \cdot 0$ to $7 \cdot 3 \%$. A total of 52 eyes missed 1 or 2 points immediately by the blind spot, and 54 eyes $(3 \cdot 13 \%, 95 \%$ CL $2 \cdot 3$ to $4 \cdot 0 \%)$ missed points elsewhere in the field.

Forty-two eyes from 33 patients (mean age 70.8 years) required an extended field as a result of non-glaucomatous disease, 31 eyes falling into the pathological category as defined above. In only three of the others was the defect limited to 1 or 2 points immediately adjacent to the blind spot.

Eyes classified as normal on all three screening parameters numbered 1510 (755 patients). The mean time taken to perform the screening programme on both eyes of these patients was 2 minutes 30 seconds. Sixty-four eyes $(4 \cdot 3 \%$, $95 \%$ CL 3.3 to $5 \cdot 3 \%$ ) from 56 patients (mean age 61.4 years) failed the field screening programme, eight patients failing in both eyes. Of the remaining 54 eyes, 16 were right eyes and 38 were left $\left(0.05>p>0.01\right.$ by $\chi^{2}$ analysis). Forty-five eyes missed only 1 or 2 points immediately adjacent to the blind spot and none of these missed other points on the 66-point extended field $(p<0.0001)$.

An eye failing the screening programme where the defect is limited to one or two misses immediately adjacent to the blind spot is therefore 11.5 times more likely to be normal than to have any demonstrable pathology. None of the eyes later confirmed as suffering from glaucoma missed spots immediately adjacent to the blind spot as the sole reason for failing the screening test.

The $\mathrm{PP}+$ of the Henson screening programme used in isolation in a population with a $1 \%$ ocular prevalence of glaucomatous field loss comprising 10000 eyes from persons aged 50 and over can be estimated as follows:

$0.9 \%$ of 10000 will fail the screen because they do have glaucoma $=$ 90 . However, $6 \cdot 15 \%$ of the 9900 non-glaucomatous eyes will also fail the screen $=609$.

$$
\mathrm{PP}+=\frac{1}{\frac{609}{90}+1} \times 100=12 \cdot 9 \% .
$$

This figure assumes any point missed at $0.5 \mathrm{log}$ unit over threshold to be significant. The $95 \%$ CL are $11 \cdot 1$ to $15 \cdot 4 \%$.

If one or two misses immediately adjacent to the blind spot are considered insignificant, the $\mathrm{PP}+$ at $1 \%$ glaucoma prevalence rises to $23 \cdot 1 \%$ (95\% CL $18 \cdot 5$ to $28 \cdot 3 \%$ ).

Figure 1 shows the manner in which the PP+ of the Henson screening programme would be expected to change in populations with increasing prevalence of glaucomatous field loss. Calculations assume no change in the prevalence of other conditions causing field loss.

Figure 2 indicates the change in $\mathrm{PP}+$ when other ophthalmoscopically recognisable conditions causing field loss are excluded.

\section{Discussion}

This study aimed to calculate the predictive power of a positive field screen on the Henson CFS2000 in a population of middle aged and 
elderly persons. The study design attempted to reduce false negatives to a minimum.

The results would appear to confirm that it is reasonable to ignore misses on the screening programme immediately adjacent to the blind spot. This would reduce the number of normal eyes in a population failing the Henson screening test from between 3.3 and $5 \cdot 3 \%$ to between 0.7 and $1.8 \%$ (applying $95 \%$ confidence limits). An isolated apparent enlargement of the blind spot on bowl perimetry was originally taken to be a sign of an early functional defect, ${ }^{3}$ but this has since been abandoned. ${ }^{+}$

It is interesting that the left eyes of normal persons were statistically more likely than right eyes to fail the screening test employed in our study. However, when misses immediately adjacent to the blind spot are removed from the analysis, the asymmetry loses its significance. This indicates that we may be observing a fatigue effect, as the left eye was always the second to be tested, small changes in fixation producing an apparent enlargement of the blind spot. Fatigue effects are common in longer psychophysical tests but would not be expected in tests of such short duration as three or four minutes. ${ }^{5}$ Moreover, fatigue would be expected to occur earlier, as age and the time taken to complete the test increase ${ }^{6}$ However, the mean age of normal persons who failed the field screen was not greater than those who passed.

Accurate refraction of all subjects was not considered feasible in a study such as ours. We therefore performed field analysis with either single vision reading spectacles or unaided, preferring to eradicate possible false positives from the edge of bifocal segments. The question of the effect of uncorrected refractive errors therefore remains.

In static perimetry, peripheral threshold sensitivity decreases linearly with increasing eccentricity, the slope of the decline being approximately parallel when increasing optical errors are simulated. ${ }^{7}$ The Henson test, in effect, compares peripheral sensitivity with predetermined paracentral thresholds, and therefore an increased paracentral threshold from uncorrected optical errors would not be expected to produce false positive or negative results in the Bjerrum areas.

Some of the screening programme failures, with points missed around the disc, may be due to uncorrected optical errors producing artificial enlargement of the blind spot. The excess of left eyes in this group indicates that, unless left eyes in our unaided subjects had significantly greater optical errors, this effect is minimal. Other factors such as the method of padding the left eye during testing of the right eye may explain this phenomenon, but these cannot be determined from the results of this study.

Our study population is known to be representative of the population of Nottingham. Despite this, care should still be taken when interpreting the results. Alterations in any of the variables may have a profound effect on the $\mathrm{PP}+$. In particular it is very dependent on a strict screening protocol being observed.

The prevalence of glaucomatous field loss is known to increase with age ${ }^{8}$ and in certain racial groups. ${ }^{9}$ Although the $\mathrm{PP}+$ will always increase with a higher population prevalence of the disease, it will not do so in a linear manner. For example other conditions producing field loss may also be more prevalent. However, an experienced, confident ophthalmoscopist will be able to increase the $\mathrm{PP}+$ of the screening programme by recognising these 'false positives.' For example, in a similar population with a glaucoma prevalence of $2 \%$, the $\mathrm{PP}+$ would rise from $23 \%$, if any miss was considered significant, to $59 \%$ if other causes of field loss are identified and misses by the blind spot are ignored (see Figs 1 and 2).

As recently as the early 1980 s $33 \%$ of glaucoma sufferers presented with advanced field loss in at least one eye. ${ }^{10}$ In a recent study of optometrists who screened for glaucoma only $8 \%$ routinely used visual field analysis. ${ }^{2}$ It has been estimated that $50 \%$ of patients with glaucomatous eyes present with an IOP within the accepted normal range. ${ }^{8}$ Moreover, the optic disc in early glaucoma is known to be notoriously difficult to assess. ${ }^{11}$ Consequently it is perhaps not surprising that patients continue to present with advanced disease. Indeed, it is not uncommon for such patients to report that they have been passed as normal by an optometrist within a few months of presenting to the hospital eye service.

Although field analysis is likely to detect only those eyes that have lost more than $50 \%$ of their nerve fibres, ${ }^{12}$ it is these eyes which require urgent attention if vision is to be preserved. ${ }^{13}$ Our study lends weight to the argument that routine central field analysis should be performed by optometrists during their glaucoma screening protocol on their middle aged and elderly clients. We have shown it can be completed quickly with acceptable predictive power levels, provided a strict protocol is observed.

The authors have no financial interest in the Henson CFS2000 field screener. This work was supported in part by grants from the International Glaucoma Association and MSD Ltd. The screening equipment used in this study was kindly loaned by Keeler Ltd.

1 Henson DB, Bryson H. Clinical results with the HensonHamblin CFS2000. Doc Ophthalmol Proc Ser 1987; 49: 233-8. Eye 1989; 3: 743-6.

3 Kahn HA, Leibowitz HM, Ganley JP, et al. The Framingham eye study 1 . Outline and major prevalence findings. Am J Epidemiol 1977; 106: 17-32.

4 Kahn HA, Milton RC. Revised Framingham eye study prevalence of glaucoma and diabetic retinopathy. $A m$ f Epidemiol 1980; 111: 769-76.

5 Keltner JL, Johnson CA. Current status of automated perimetry. Is the ideal perimeter available? Arch Ophthalmol 1986; 104: 347-9.

6 Kosodo O, Sommer A, Auer C. Duration of automated suprathreshold vs quantitative threshold field examination. Arch Ophthalmol 1986; 104: 398-401.

7 Heuler DA, Anderson DR, Feuer WJ, Gressel MG. The influence of refraction accuracy on automated perimetric influence of refraction accuracy on automated perimetric
threshold measurements. Ophthalmology 1987; 94: 1550-3.

8 Hollows FC, Graham PA. Intraocular pressure, glaucoma, and glaucoma suspects in a defined population. Brf Ophthalmol $1966 ; 50: 570-86$

9 Leske MC, Connell AMS, Kehoe R. A pilot project of glaucoma in Barbados. Brf Ophthalmol 1989; 73: 365-9.

10 Elkington AR, Lewry J, MacKean J, Sargent P. A collaborative hospital glaucoma survey. Res Clin Forums 1982; 4:(5) $31-40$

11 Pederson JE, Anderson DR. The mode of progressive disc cupping in ocular hypertension and glaucoma. Arch Ophthalmol 1980; 98: 490-5.

12 Quigley HA, Addicks EM, Green WR. Optic nerve damage in human glaucoma III. Quantitative correlation of nerve fibre loss and visual field defect in glaucoma, ischaemic optic neuropathy, papilledema and toxic neuropathy. Arch Oph thalmol 1982; 100: 135-46.

13 Grant WM, Burke JF. Why do some people go blind from glaucoma? Ophthalmology 1982; 89: 991-8. 